\title{
Beyond genus statistics: a unifying approach to the morphology of cosmic structure
}

\author{
Jens Schmalzing ${ }^{1,2, *} \quad$ Thomas Buchert ${ }^{1, \dagger}$ \\ submitted to Ap. J. Lett.
}

\begin{abstract}
The genus statistics of isodensity contours has become a well-established tool in cosmology. In this Letter we place the genus in the wider framework of a complete family of morphological descriptors. These are known as the Minkowski functionals, and we here apply them for the first time to isodensity contours of a continuous random field. By taking two equivalent approaches, one through differential geometry, the other through integral geometry, we derive two complementary formulae suitable for numerically calculating the Minkowski functionals. As an example we apply them to simulated Gaussian random fields and compare the outcome to the analytically known results, demonstrating that both are indeed well suited for numerical evaluation. The code used for calculating all Minkowski functionals is available from the authors.
\end{abstract}

\section{Introduction}

The ambitious goal of characterizing the morphology of cosmic structure in a unique and complete way leads to the field of integral geometry. There, this problem has been well-posed and Hadwiger's theorem (Hadwiger 1957, see also Klain 1995 for a proof) gives a powerful answer: In $d$ spatial dimensions the global morphological properties (defined as those which satisfy motional invariance and additivity) of any pattern can be completely characterized by $d+1$ numbers, the so-called Minkowski functionals (Minkowski 1903). Furthermore, in the interesting cases of two and three dimensions, all the Minkowski functionals can be intuitively interpreted in terms of well-known geometric quantities.

One of them is the Euler characteristic $\chi$, or equivalently the genus $g=1-\chi$, which has long been used as a statistical tool in cosmology. The standard analysis technique starts from a continuous field - a density or temperature field. For a point process this is usually constructed by applying a smoothing filter with a constant width larger than the mean separation of points. For this field, the Euler characteristic of an isocontour at level $\nu$ is obtained, according to the GaussBonnet theorem, through a surface integration of the Gaussian curvature. This threshold level can be employed as a diagnostic parameter, and the genus represented as a function of $\nu$ (see Weinberg et al. 1987, Melott 1990, Coles et al. 1996 and references therein). This quantity is known analytically for Gaussian random fields (Doroshkevich 1970) and even for some nonlinear dynamical models such as solutions of the Lagrangian perturbation theory Matsubara \& Suto 1996; Seto et al. 1997).

This method for calculating the genus or, as advocated in the present Letter, all four Minkowski functionals, suffers from erasing small-scale information (ter Haar Romeny et al. 1991) when going from point sets to smooth density fields. While this may actually be an advantage when

\footnotetext{
${ }^{1}$ Ludwig-Maximilians-Universität, Theresienstraße 37, 80333 München, Germany

${ }^{2}$ Max-Planck-Institut für Astrophysik, Karl-Schwarzschild-Straße 1, 85740 Garching, Germany

*email jensen@mpa-garching.mpg.de

†email buchert@stat.physik.uni-muenchen.de
} 
trying to deemphasize poorly understood effects such as nonlinear evolution and biasing, some discriminatory power is lost. However, one may think of different methods of smoothing which retain more information, for example varying the smoothing length according to the local density. In this situation, Mecke et al. (1994) introduced Minkowski functionals of point processes into cosmology. Proceeding as directly as possible they decorate each point with a ball of a certain radius $R$, which becomes the diagnostic parameter for this method of analysis. Thereby a union set of balls is constructed and probes the clustering properties of the point set. As stated above, these morphological properties can be measured in a complete and unique way using Minkowski functionals, so powerful methods of integral geometry (Weil 1983) become applicable. Most notably, Minkowski functionals contain a variety of other descriptors such as the "void probability function" (White 1979), and depend on the complete hierarchy of correlations (Stratonovich 1963; Mecke et al. 1994). Analytically known results for the Poisson process (Mecke \& Wagner 1991) provide a useful standard of reference; the additivity property in integral geometry leads to robustness against various sources of error (Kerscher et al. 1997a); the sensitivity to small-scale clustering properties allows for efficient discrimination, as has been demonstrated in recent applications to the Abell/ACO cluster catalogue in comparison with mock catalogues drawn from simulations (Kerscher et al. 1997b) and the fluctuations in the IRAS 1.2Jy catalogue (Kerscher et al. 1997a). A brief tutorial on Minkowski functionals in cosmology can be found in Schmalzing et al. (1995). In this Letter we advocate the use of the whole family of Minkowski functionals to analyze the continuous random fields encountered in cosmology. Thereby we obtain more information than by measuring the genus alone. Furthermore, recent work (especially Kerscher et al. 1997a, see also Buchert 1995 for a direct comparison) has shown that other Minkowski functionals such as the surface area and the integrated mean curvature can possess greater discriminatory power than the Euler characteristic. Apart from this practical advantage, which was already pointed out for the area by Ryden et al. (1989), the use of all the Minkowski functionals leads to a conceptual generalization of genus statistics, incorporates it into the firm mathematical framework of integral geometry and stereology and allows for a straightforward evaluation, including exact correction for boundary effects.

The Letter is organized as follows. Section 2 presents an approach to Minkowski functionals of density fields that uses the methods of differential geometry to reduce the surface integration to a spatial average of Koenderink invariants. Section 3 employs integral geometry, namely Crofton's intersection formula, to derive a second calculational method that relies on the representation of the density field by its values at lattice points. Section 4 gives the analytical formulae found by Tomita (1990) for all the Minkowski functionals of Gaussian random fields, while Section 5 compares them to the results obtained by applying our two approximation formulae to numerically simulated Gaussian random fields. Section 6 provides an outlook. Further applications are postponed to subsequent papers.

\section{Koenderink Invariants}

Consider a random field $\nu(\mathbf{x})$ on a $d$-dimensional support $\Omega \subseteq \mathbb{R}^{d}$. For a given threshold $\nu$ the excursion set $F_{\nu}$ is the set of all points $\mathbf{x}$ with $\nu(\mathbf{x}) \geq \nu$. We wish to calculate its Minkowski functionals $\mathrm{v}_{k}^{(d)}(\nu)$ per unit volume. The volume functional $\mathrm{v}_{0}^{(d)}$ is simply given by volume integration of a Heaviside step function $\Theta$, normalized to the whole volume $|\Omega|$,

$$
\mathrm{v}_{0}^{(d)}(\nu)=\frac{1}{|\Omega|} \int_{\Omega} \mathrm{d}^{d} x \Theta(\nu-\nu(\mathbf{x})) .
$$

In three dimensions, the other Minkowski functionals correspond to quantities known from differential geometry, namely the surface area, the integrated mean curvature and the Euler characteristic (Schneider 1993). They can be evaluated by a two-dimensional surface integration

$$
\mathrm{v}_{k}(\nu)=\frac{1}{|\Omega|} \int_{\partial F_{\nu}} \mathrm{d}^{2} A(\mathbf{x}) \mathrm{v}_{k}^{(\text {loc })}(\nu, \mathbf{x})
$$


The integrands

$$
\begin{aligned}
& \mathrm{v}_{1}^{(\text {loc })}(\nu, \mathbf{x})=\frac{1}{6} \\
& \mathrm{v}_{2}^{(\text {loc })}(\nu, \mathbf{x})=\frac{1}{6 \pi}\left(\frac{1}{R_{1}}+\frac{1}{R_{2}}\right) \\
& \mathrm{v}_{3}^{(\text {loc })}(\nu, \mathbf{x})=\frac{1}{4 \pi} \frac{1}{R_{1} R_{2}}
\end{aligned}
$$

are nothing but the three invariants made from the inverse radii of curvature $R_{1}$ and $R_{2}$ of the surface - with signs chosen to reflect the orientation towards lower density values. The normalization factors yield the conventional definition of the Minkowski functionals.

Using methods developed by Koenderink (Koenderink 1984; ter Haar Romeny et al. 1991) in twodimensional image analysis, it is possible to express these local curvatures in terms of geometric invariants formed from first and second derivatives (Schmalzing 1996). However, the surface integration (2) of these invariants is equivalent to taking the spatial mean over the whole volume, with a gradient for the transformation to the surface element and a delta function for selecting the isodensity contour added as factors. The resulting formula is

$$
\mathrm{v}_{k}(\nu)=\frac{1}{|\Omega|} \int_{\Omega} \mathrm{d}^{3} x \delta(\nu-\nu(\mathbf{x}))|\nabla \nu(\mathbf{x})| \mathrm{v}_{k}^{(\text {loc })}(\nu, \mathbf{x}) .
$$

If the density field is sampled at the points of some lattice, we can therefore estimate the Minkowski functionals by calculating the derivatives at each lattice point and replacing first the $\delta$-function with a bin of finite width and then the average over all space with a summation over lattice points only. Analogous formulae can be derived in two dimensions, so the most promising cosmological application of this particular method is an elegant way of calculating Minkowski functionals for fields on the sphere, like all-sky maps of the CMB (for existing work, see Smoot et al. 1994, Colley et al. 1996 and especially Torres et al. 1995).

\section{Crofton's Intersection Formula}

Another powerful approach to morphology of density fields comes through integral geometry (Hadwiger 1957: Santaló 1976). As above we consider a random field $\nu(\mathbf{x})$ and measure the Minkowski functionals $\mathrm{v}_{k}^{(d)}(\nu)$ of its excursion set over a threshold $\nu$.

To derive an approximation formula we use Crofton's formula (Crofton 1868) which relates the Minkowski functionals from different dimensions. For a body $K$ in $d$ dimensions we consider an arbitrary $k$-dimensional hyperplane $E$ and calculate the Euler characteristic $\chi$ of the intersection $K \cap E$ in $k$ dimensions. Integrating this quantity over the space $\mathcal{E}_{k}^{(d)}$ of all conceivable hyperplanes円 we obtain the $k$ th Minkowski functional of the body $K$ in $d$ dimensions. Crofton's formula states that t $^{2}$

$$
\mathrm{v}_{k}^{(d)}(K)=\frac{\omega_{d}}{\omega_{d-k} \omega_{k}} \int_{\mathcal{E}_{k}^{(d)}} \mathrm{d} \mu_{k}(E) \chi^{(k)}(K \cap E)
$$

If the body $K$ is the excursion set of a homogeneous and isotropic random field sampled at $L$ points of a cubic lattice of spacing $a$, we can as well average over the set $\mathcal{L}_{k}^{(d)}$ of all lattice hyperplanes only. The integral is then replaced by a summation over these hyperplanes with an additional normalization factor and we obtain

$$
\mathrm{v}_{k}^{(d)}(K)=\frac{\omega_{d}}{\omega_{d-k} \omega_{k}} \sum_{E \in \mathcal{L}_{k}^{(d)}} \frac{1}{a^{k} L} \frac{k !(d-k) !}{d !} \chi^{(k)}(K \cap E) .
$$

\footnotetext{
${ }^{1}$ The integration measure $\mathrm{d} \mu_{k}(E)$ is normalized to give $\int_{\mathcal{E}_{k}^{(d)}} \mathrm{d} \mu_{k}(E)=1$.

${ }^{2} \omega_{k}$ is the volume of the unit ball in $k$ dimensions, so $\omega_{0}=1, \omega_{1}=2, \omega_{2}=\pi$ and $\omega_{3}=\frac{4}{3} \pi$.
} 
However, the Euler characteristic of a body in $k$ dimensions can be approximated by counting the numbers $N_{j}(K)$ of all elementary lattice cells of dimension $j$ within the body (Adler 1981). In three dimensions, for example, $N_{3}(K)$ gives the number of cubes within the body, $N_{2}(K)$ and $N_{1}(K)$ are the numbers of faces and edges of the lattice cubes, respectively, while $N_{0}(K)$ gives the number of lattice points contained in $K$. In general we have

$$
\chi^{(k)}(K \cap E)=\sum_{j=0}^{k}(-1)^{j} N_{j}(K \cap E) .
$$

When summing equation (7) over all lattice hyperplanes, we notice that $\left(\begin{array}{c}d-j \\ k-j\end{array}\right) k$-dimensional hyperplanes $E$ pass through each $(d-k+j)$-dimensional lattice cell belonging to the body $K$, and the intersection is a $j$-dimensional lattice cell of the body $K \cap E$. Therefore, we can actually perform the summation

$$
\sum_{E \in \mathcal{L}_{k}^{(d)}} N_{j}(K \cap E)=\left(\begin{array}{c}
d-j \\
k-j
\end{array}\right) N_{d-k+j}(K)
$$

and end up with the compact formula

$$
\mathrm{v}_{k}^{(d)}(K)=\frac{\omega_{d}}{\omega_{d-k} \omega_{k}} \frac{1}{a^{k} L} \sum_{j=0}^{k}(-1)^{j} \frac{k !(d-j) !}{d !(k-j) !} N_{d-k+j}(K) .
$$

For a homogeneous and isotropic random field in three dimensions the Minkowski functionals are thus given by

$$
\begin{aligned}
\mathrm{v}_{0}(K) & =\frac{1}{L} N_{3}(K) \\
\mathrm{v}_{1}(K) & =\frac{1}{a L}\left(-\frac{2}{9} N_{3}(K)+\frac{2}{3} N_{2}(K)\right) \\
\mathrm{v}_{2}(K) & =\frac{1}{a^{2} L}\left(\frac{2}{9} N_{3}(K)-\frac{4}{9} N_{2}(K)+\frac{2}{3} N_{1}(K)\right) \\
\mathrm{v}_{3}(K) & =\frac{1}{a^{3} L}\left(-N_{3}(K)+N_{2}(K)-N_{1}(K)+N_{0}(K)\right) .
\end{aligned}
$$

Note that the last relation for the Euler characteristic $\mathrm{v}_{3}=\chi /|\Omega|$ per unit volume is not obtained from Crofton's formula but is already given as a special case of equation (7) which results from the Morse theorem (Morse \& Cairns 1969). Being conceptually simple in comparison to the other approximations, it was already proved rigorously by Adler (1981) and used by Coles et al. (1996) to calculate the genus of isodensity contours in three dimensions. Adding Crofton's formula gives all the Minkowski functionals without further computational effort, since the calculation requires nothing but the counting of all elementary lattice cells, which is already necessary for calculating the genus.

\section{Tomita's Formulae for Statistics of Random Interfaces}

Just like the genus (Doroshkevich 1970), all Minkowski functionals are analytically known for a Gaussian random field, due to a highly instructive article by Tomita (1990) which investigates a Gaussian random field $\nu(\mathbf{x})$ in $d$ dimensions. Again, let us consider the excursion set over a certain threshold $\nu$ and calculate its mean Minkowski functionals $\mathrm{v}_{k}^{(d)}(\nu)$ per unit volume.

It turns out that the result depends on only two parameters $\sigma$ and $\lambda$, which in turn depend on the value of the correlation function $\xi(0)$ and its second derivative $\xi^{\prime \prime}(0)$ at zero through

$$
\sigma=\xi(0), \quad \lambda=\sqrt{\left|\xi^{\prime \prime}(0)\right| /(2 \pi \xi(0))} .
$$


Both are easily measured from a given realization of the random field, since $\xi(0)=\left\langle\nu^{2}\right\rangle$ is the variance of the field itself and $\left|\xi^{\prime \prime}(0)\right|=\left\langle\nu_{, i}^{2}\right\rangle$ is the variance of any of its first derivatives (Adler 1981 .

With these two parameters all Minkowski functionals in arbitrary dimension $d$ can be calculated for a Gaussian random field (Tomita 1990). For the volume functional we obtain?

$$
\mathrm{v}_{0}^{(d)}(\nu)=\frac{1}{2}-\frac{1}{2} \Phi\left(\frac{\nu}{\sqrt{2 \sigma}}\right)
$$

while all other Minkowski functionals are given by

$$
\mathrm{v}_{k}^{(d)}(\nu)=\lambda^{k} \frac{\omega_{d}}{\omega_{d-k} \omega_{k}} H_{k-1}\left(\frac{\nu}{\sqrt{\sigma}}\right) .
$$

Putting $d=3$ we obtain the Minkowski functionals in three dimensions

$$
\begin{aligned}
& \mathrm{v}_{0}(\nu)=\frac{1}{2}-\frac{1}{2} \Phi\left(\frac{1}{\sqrt{2}} u\right) \\
& \mathrm{v}_{1}(\nu)=\frac{2}{3} \frac{\lambda}{\sqrt{2 \pi}} \exp \left(-\frac{1}{2} u^{2}\right) \\
& \mathrm{v}_{2}(\nu)=\frac{2}{3} \frac{\lambda^{2}}{\sqrt{2 \pi}} u \exp \left(-\frac{1}{2} u^{2}\right) \\
& \mathrm{v}_{3}(\nu)=\frac{\lambda^{3}}{\sqrt{2 \pi}}\left(u^{2}-1\right) \exp \left(-\frac{1}{2} u^{2}\right) .
\end{aligned}
$$

In the next section we will compare these analytical values to the values obtained by numerical evaluation using the formulae (4) and (10).

\section{Gaussian Random Fields}

Gaussian random fields provide a useful standard of reference and are always good for a pedagogical example. Here we use a scale-invariant power spectrum to construct Gaussian random fields on $128^{3}$ points in a unit cube. We apply a Gaussian smoothing filter of width $16 / 128=0.125$, which is chosen large in order to give a large variation between realizations. The field is normalized to $\langle\nu\rangle=0$ and $\left\langle\nu^{2}\right\rangle=1$. Then, we calculate the numerical estimates and the analytical predictions at 100 threshold values between -4 and 4 and obtain the mean and variance over 200 such curves. Figure 1 shows three areas in each plot. One is measured by averaging Koenderink invariants as in equation (14), the second by using Crofton's formula (10). However, these two are completely indistinguishable, which shows that the two approximations are indeed equivalent.

A third area (shaded, dotted lines) is obtained by estimating the parameters $\sigma$ and $\lambda$ from each random field and computing the analytical mean Minkowski functionals according to (14). Obviously the measured mean values fit the analytical expectations extremely well; deviations are far from significant. Since the analytical curves incorporate only statistical fluctuations in the determination of the parameters, their variance is reduced compared to the measured curves, which contain the full variance between and within samples. Obviously, surface area $\mathrm{v}_{1}$ and integrated mean curvature $\mathrm{v}_{2}$ vary almost exclusively through parameter fluctuations, so these measures enhance the discriminatory power compared to considering the Euler characteristic $\mathrm{v}_{3}$ alone.

\section{Summary and Outlook}

We have extended the scope of genus statistics by considering all four Minkowski functionals in three dimensions. Two independent approaches gave us two different approximation formulae

\footnotetext{
${ }^{3} \Phi(x)=\frac{2}{\sqrt{\pi}} \int_{0}^{x} \mathrm{~d} t \exp \left(-t^{2}\right)$ denotes the Gaussian error integral, while $H_{n}(x)=\left(-\frac{\mathrm{d}}{\mathrm{d} x}\right)^{n} \frac{1}{\sqrt{2 \pi}} \exp \left(-\frac{1}{2} x^{2}\right)$ defines a Hermite function of order $n$.

${ }^{4}$ To relieve the notation of the overall normalization $\sqrt{\sigma}$, we use the dimensionless argument $u$, with $\nu=u \sqrt{\sigma}$.
} 

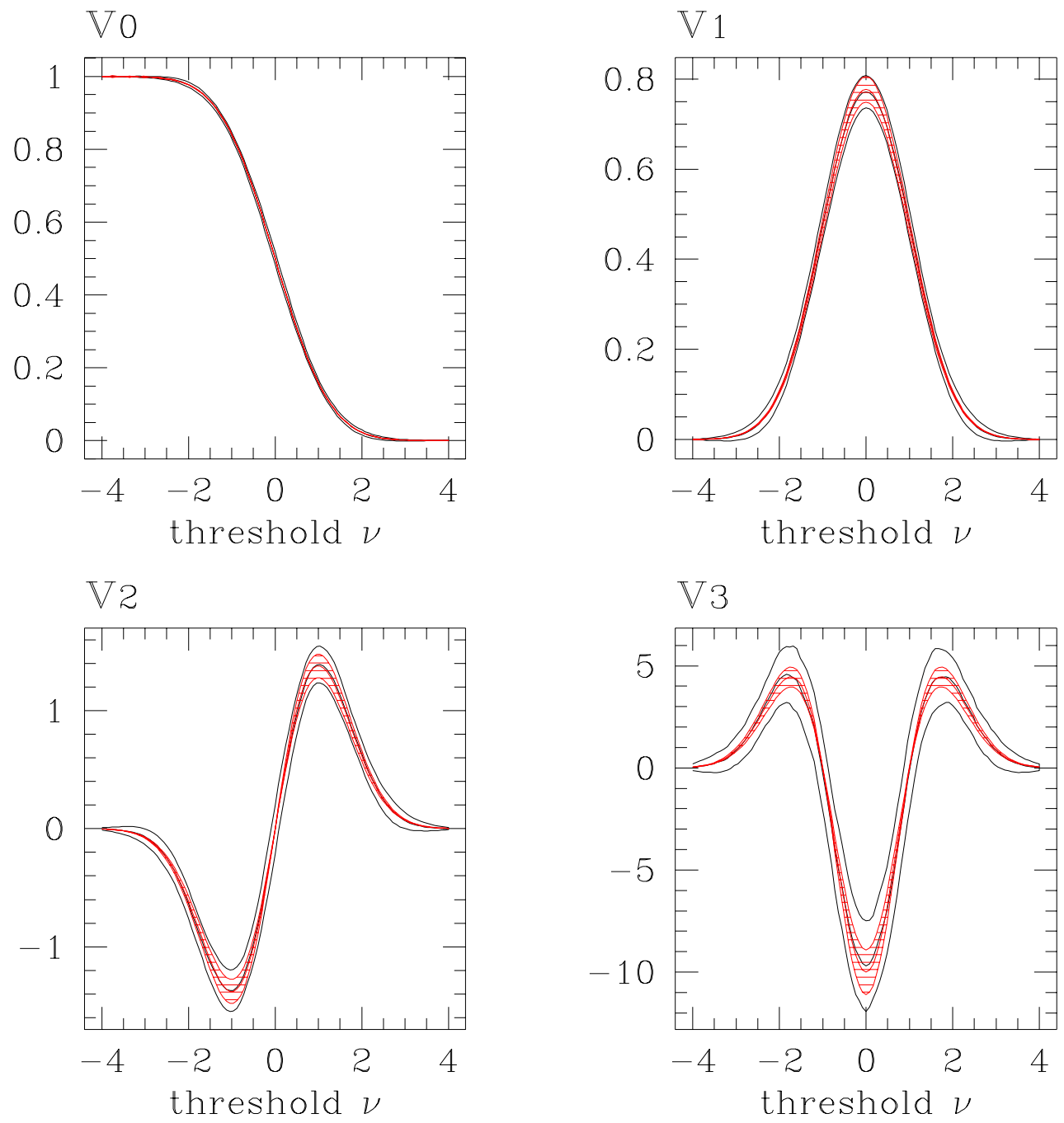

Figure 1: The four Minkowski functionals of isodensity contours of a Gaussian random field in three dimensions. The central lines show the exact mean values while the area between top and bottom lines indicates the statistical variance of our results over 200 realizations. Actually there are three areas in each plot, corresponding to the three formulae (4), (10) and (14). However, the two independent approximation formulae (no shading) perform so well that their results are identical. Also, the agreement with analytically predicted mean values (shaded) is remarkable. 
which we have tested by application to a Gaussian random field. Furthermore, we verified that our measurements fit the analytically known Minkowski functionals of a Gaussian random field. Even when analytical reference values are not known, we have two complementary numerical methods at our disposal which both rely on the approximation of a smooth density field by values at lattice points. The agreement between the two can be employed to determine the necessary smoothing length. Too little smoothing would be easily detectable by discrepancies between the two methods.

Forthcoming applications of the presented method will give intuitive interpretations of our novel access to cosmic morphology. Also, issues such as boundary corrections (see Kerscher et al. 1996 for point processes) and other problems which arise for observational data have to be addressed. Further tests of the robustness and discriminatory power are necessary beyond the promising results given here.

With the methodical and theoretical framework laid out, we can also tackle the application of our method both to large-scale structure and to smaller entities such as individual clusters of galaxies. Quantitative measurements on the network of large-scale structure are definitely within reach. It is important to use this new method on both real and simulated data. The broad and unified approach promises significant insights into the nature and evolution of the largest structures in our Universe.

\section{Acknowledgements}

We thank Claus Beisbart, Martin Kerscher, Adrian Melott, Herbert Wagner, David Weinberg, and especially Simon White for interesting discussions and valuable comments. TB acknowledges support by the "Sonderforschungsbereich 375 für Astroteilchenphysik der Deutschen Forschungsgemeinschaft".

For a continuous random field sampled on a cubic lattice the code for calculating all Minkowski functionals as functions of threshold can be obtained from the authors.

\section{REFERENCES}

Adler, R. J., The geometry of random fields, John Wiley \& Sons, Chichester, 1981.

Buchert, T., in: 11th Potsdam Workshop on Large-Scale Structure in the Universe (Geltow) (Mücket, J., Gottlöber, S., \& Müller, V., eds.), World Scientific, 1995, pp. 156-161.

Coles, P., Davies, A., \& Pearson, R. C., Mon. Not. R. Astron. Soc. 281 (1996), 1375-1384.

Colley, W. N., Gott III, J. R., \& Park, C., Mon. Not. R. Astron. Soc. 281 (1996), L82-L84.

Crofton, M. W., Phil. Trans. Roy. Soc. London 158 (1868), 181-199.

Doroshkevich, A. G., Astrophysics 6 (1970), 320-330.

Hadwiger, H., Vorlesungen über Inhalt, Oberfläche und Isoperimetrie, Springer Verlag, Berlin, 1957.

Kerscher, M., Schmalzing, J., \& Buchert, T., in: Mapping, measuring and modelling the universe

(Valencia) (Coles, P., Martínez, V., \& Pons Bordería, M. J., eds.), Astronomical Society of the Pacific, 1996, pp. 247-252.

Kerscher, M., Schmalzing, J., Buchert, T., \& Wagner, H., to be submitted, 1997.

Kerscher, M., Schmalzing, J., Retzlaff, J., Borgani, S., Buchert, T., Gottlöber, S., Müller, V., Plionis, M., \& Wagner, H., Mon. Not. R. Astron. Soc. 284 (1997), 73-84.

Klain, D. A., Mathematika 42 (1995), 329-339.

Koenderink, J. J., Biol. Cybern. 50 (1984), 363-370.

Matsubara, T. \& Suto, Y., Ap. J. 460 (1996), 51-58.

Mecke, K. \& Wagner, H., J. Stat. Phys. 64 (1991), 843.

Mecke, K., Buchert, T., \& Wagner, H., Astron. Astrophys. 288 (1994), 697.

Melott, A. L., Physics Rep. 193 (1990), 1-39.

Minkowski, H., Mathematische Annalen 57 (1903), 447-495. 
Morse, M. \& Cairns, S. S., Critical point theory in global analysis and differential topology, Academic Press, New York and London, 1969.

Ryden, B. S., Melott, A. L., Craig, D. A., Gott III, J. R., Weinberg, D. H., Scherrer, R. J., Bhavsar, S. P., \& Miller, J. M., Ap. J. 340 (1989), 647-660.

Santaló, L. A., Integral Geometry and Geometric Probability, Addison-Wesley, Reading, MA, 1976.

Schmalzing, J., Kerscher, M., \& Buchert, T., in: Proceedings of the international school of physics Enrico Fermi. Course CXXXII: Dark matter in the universe (Bonometto, S., Primack, J., \& Provenzale, A., eds.), Societá Italiana di Fisica, 1995.

Schmalzing, J., Diplomarbeit, Ludwig-Maximilians-Universität München, 1996, in German, English excerpts available.

Schneider, R., Convex bodies: the Brunn-Minkowski theory, Cambridge University Press, Cambridge, 1993.

Seto, N., Yokoyama, J., Matsubara, T., \& Siino, M., Ap. J. Suppl. 110 (1997).

Smoot, G. F., Tenorio, L., Banday, A. J., Kogut, A., Wright, E. L., Hinshaw, G., \& Bennett, C. L., Ap. J. 437 (1994), 1-11.

Stratonovich, R. L., Topics in the theory of random noise, Vol. 1, Gordon and Breach, New York, 1963.

ter Haar Romeny, B. M., Florack, L. M. J., Koenderink, J. J., \& Viergever, M. A., in: Lecture Notes in Computer Science, Vol. 511, Springer Verlag, Berlin, 1991, pp. 239-255.

Tomita, H., in: Formation, dynamics and statistics of patterns (Kawasaki, K., Suzuki, M., \& Onuki, A., eds.), Vol. 1, World Scientific, 1990, pp. 113-157.

Torres, S., Cayón, L., Martínez-González, E., \& Sanz, J. L., Mon. Not. R. Astron. Soc. 274 (1995), 853-857.

Weil, W., in: Convexity and its applications (Gruber, P. M. \& Wills, J. M., eds.), Birkhäuser, Basel, 1983, pp. 360-412.

Weinberg, D. H., Gott III, J. R., \& Melott, A. L., Ap. J. 321 (1987), 2-27.

White, S. D. M., Mon. Not. R. Astron. Soc. 186 (1979), 145-154. 